\title{
Research on the Transfer Characteristics of the Micro Particle in a Corrugated Pipe
}

\author{
Ding Yu, Ning Zhi ${ }^{*}$ Lü Ming \\ School of Mechanical, Electronic and Control Engineering, Beijing Jiaotong University, Beijing, China \\ Email address: \\ zhining@bjtu.edu.cn (Ning Zhi) \\ ${ }^{*}$ Corresponding author \\ To cite this article: \\ Ding Yu, Ning Zhi, Lü Ming. Research on the Transfer Characteristics of the Micro Particle in a Corrugated Pipe. International Journal of \\ Economy, Energy and Environment. Vol. 4, No. 4, 2019, pp. 71-79. doi: 10.11648/j.ijeee.20190404.12
}

Received: June 21, 2019; Accepted: August 3, 2019; Published: August 27, 2019

\begin{abstract}
The corrugated pipe is widely used in figure heat exchangers. For the reason of large temperature difference between the gas in the pipe and the cold wall, particles may deposit on the wall, and make some adverse effects, such as poor cooling effect and the block in the pipe, especially in the pipe node. To solve the problem of deposition in the pipe, this paper will apply the model of DRW to calculate the trajectory of the micro particles. In this paper, the trajectories of particles with different sizes in a corrugated pipe are calculated, and the effects of structure parameter and inlet velocity on the trajectories of particles are studied. The conclusion can provide a theoretical basis for solving the deposition in corrugated pipes.
\end{abstract}

Keywords: Corrugated Pipe, Micro Particles, DPM Model

\section{Introduction}

Particle movement in the turbulent channel is a typical problem of gas-solid flow. There are many forms of exercise of particles in a turbulent channel such as following the fluid, settling to the wall, suspending from the wall and so on. In many cases, the deposition of particles in the channel is the most common phenomenon and may cause adverse effects like jamming the channel, so most of the studies on this subject are about the transport and deposition of the particles. As early as in 1984, there were many scientists like Wood, Hinds, Hidy and Papavergos studying on the motion of particles in turbulent flow by theoretical and experimental method [1-3].

Initially scientists like McLaughlin, Ahmadi and Thakurta mostly took the method of direct numerical simulation and some valuable conclusions were acquired [4-6]. With the progress of the numerical simulation method, some of the gas-solid flow simulation methods became more popular, especially the Discrete Random Walk model proposed by Gosman and some other scientists, which took the influence of turbulent fluctuation into consideration [7]. This model considers the effect of turbulent fluctuation by simulating particle interaction with turbulent vortex cloud and the instantaneous velocity of the vortex cloud consists of average velocity $\bar{u}$ and fluctuation velocity $u$. Kallio and Reeks simulated the particle settlement in a vertical pipe, which took the influence of the fluid near the wall into consideration [8]. Hao $\mathrm{Lu}$ and Lin $\mathrm{Lu}$ used the modified RSM model and Lagrange method to calculate the deposition of particles in smooth and ribbed pipelines and the results showed that the internal obstructions significantly enhanced the deposition [9]. Bai Zhenxiao studied on the motion characteristics of spherical and ellipsoidal particles in turbulent passage and the result showed that for ellipsoidal particles, it is easier for them to sink down to the wall with a larger half-axis ratio [10].

It is worth noting that the previous researches on this subject were almost all based on a straight round pipe. Actually, there were little relative researches on other shapes of channel, for example the corrugated pipe. In a lot of equipment, especially in a heat exchanger, the application of the corrugated pipe is very extensive. Corrugated pipe can increase the area of heat transfer and strengthen the disturbance near the wall. But at the same time, the deposition in a corrugated pipe is especially serious for the reason of special structure and large temperature difference between the fluid and the wall. The deposition will weaken the effect of heat transfer and even result in pipe jamming. So it is significant to work on the particle transfer in a corrugated pipe.

In this paper, transfer characteristic of the particle in a corrugated pipe has been simulated numerically based on the 
Discrete Random Walk (DRW) model using the CFD simulation software ANSYS FLUENT. The governing equations and DRW method are given in Sec. 2. The solvability conditions and validation of the numerical method are presented in Sec. 3. The results and discussions are presented in Sec. 4, including the trajectories of particles and effects of particle trajectories. Conclusions are given in Sec. 5.

\section{Numerical Methods}

\subsection{Governing Equations}

Realizable $k-\varepsilon$ turbulence models are applied to simulate the flow and heat transfer in the corrugated tube. It should be pointed out that the following equations are based on the assumption that the fluid is incompressible and has constant viscosity.

Continuity equation:

$$
\frac{\partial u_{i}}{\partial x_{i}}=0
$$

Momentum equation (Navier-Stokes Equation):

$$
\frac{\partial u_{i} u_{j}}{\partial x_{i}}=-\frac{\partial p}{\rho \partial x_{i}}+\frac{\partial}{\partial x_{j}}\left[\left(\mu+\mu_{t}\right)\left(\frac{\partial u_{j}}{\partial x_{i}}+\frac{\partial u_{i}}{\partial x_{j}}\right)\right]
$$

Energy equation:

$$
\frac{\partial u_{i} T}{\partial x_{i}}=\rho \frac{\partial}{\partial x_{i}}\left[\left(\frac{\mu}{P r}+\frac{\mu_{t}}{P r_{t}}\right) \frac{\partial T}{\partial x_{i}}\right]
$$

Turbulent kinetic energy $k$ equation:

$$
\frac{\partial u_{i} k}{\partial x_{i}}=\frac{\partial}{\partial x_{i}}\left(\left(\mu+\frac{\mu_{t}}{\sigma_{k}}\right) \frac{\partial k}{\partial x_{i}}\right)+\Phi-\varepsilon
$$

Rate of turbulent kinetic energy dissipation $\varepsilon$ equation:

$$
\frac{\partial u_{j} \varepsilon}{\partial x_{j}}=\frac{\partial}{\partial x_{i}}\left(\left(\mu+\frac{\mu_{t}}{\sigma_{\varepsilon}}\right) \frac{\partial \varepsilon}{\partial x_{i}}\right)+c_{1} \Phi \varepsilon-c_{2} \frac{\varepsilon^{2}}{k+\sqrt{\mu \varepsilon}}
$$

The coefficients in $k-\varepsilon$ turbulence model are given in the following way:

$$
\begin{gathered}
\Phi=\mu_{t}\left(\frac{\partial u_{i}}{\partial x_{j}}+\frac{\partial u_{j}}{x_{i}}\right) \frac{\partial u_{i}}{\partial x_{i}} \\
\mu_{t}=\rho C_{\mu} \frac{k^{2}}{\varepsilon}
\end{gathered}
$$

Here, $\mu_{t}$ is the turbulent viscosity. $\sigma_{k}$ and $\sigma_{\varepsilon}$ are the turbulent Prandtl numbers for $k$ and $\varepsilon$, $c_{1}=\max \left[0.43, \mu /\left(\mu_{t}+5\right)\right], c_{2}=1.9, \sigma_{k}=1.0, \sigma_{\varepsilon}=1.2$.

The difference between the realizable $k-\varepsilon$ model and the standard and RNG $k-\varepsilon$ models is that $C_{\mu}$ is no longer constant and $C_{\mu}$ is a function of the mean strain and rotation rates, the angular velocity of the system rotation, and the turbulence fields ( $k$ and $\varepsilon$ ). So the Realizable $k$ - $\varepsilon$ model performs better than the Standard $k-\varepsilon$ model when calculating the flow with a rotation.

\subsection{DRW Model}

The DRW model regards the fluid phase as a continuum and the particle as a dispersed phase. It means that the fluid phase is dealt with in Euler coordinates while the particle is calculated in Lagrangian coordinates. In general, the volume fraction of the dispersed phase should be less than 5\% while the DRW model assumes that there is no collision or interference between particles.

Newton's second law equates the particle inertia with the forces acting on the particle, and the particle trajectory can be computed by the integral of the instantaneous velocity.

$$
\begin{gathered}
m_{p} \frac{\mathrm{d} u_{p}}{\mathrm{~d} t}=\Sigma F \\
u_{p}=\frac{\mathrm{d} x}{\mathrm{~d} t}
\end{gathered}
$$

\subsubsection{Force Analysis}

At first, force analysis of the particle is necessary in order to obtain the particle trajectory.

(1) Drag Force

Viscosity of the fluid results in different pressure and shear stress on the particle surface. The uneven distribution leads to drag force on particles [11].

$$
\begin{gathered}
F_{d}=\frac{1}{\tau_{r}}\left(u_{f}-u_{p}\right) \\
\tau_{r}=\frac{\rho_{p} D_{p}^{2}}{18 \mu} \frac{24}{C_{D} R e_{p}} \\
\operatorname{Re}_{p}=\frac{\rho D_{p}\left|u_{p}-u_{f}\right|}{\mu}
\end{gathered}
$$

Here, $F_{d}$ is the drag force per unit particle mass, $\tau_{r}$ is the particle relaxation time, $\rho_{p}$ is the density of the particle, $\rho$ is the fluid density, $D_{p}$ is the particle diameter, $\mu$ is the molecular viscosity of the fluid, $C_{D}$ is called as drag coefficient, $R e_{p}$ is the relative Reynolds number. $C_{D}$ is a function of $R e_{p}$, which is defined as

$$
\begin{array}{rr}
C_{D}=\frac{24}{\operatorname{Re}_{p}} & \operatorname{Re}_{p}<1 \\
C_{D}=\frac{24}{\operatorname{Re}_{p}}\left(1+0.15 \operatorname{Re}_{p}^{0.667}\right) & 1 \leq \operatorname{Re}_{p}<400 \\
C_{D}=0.44 & \operatorname{Re}_{p} \geq 400
\end{array}
$$

For micron and submicron particles in a tube, the drag force can be indicated as 


$$
\begin{gathered}
F_{d}=\frac{18 \mu}{\rho_{p} d_{p}^{2} C_{c}} \\
C_{c}=1+K_{n}\left(1.257+0.4 e^{\frac{-1.1}{K_{n}}}\right) \\
K_{n}=\frac{2 \lambda}{d_{p}}
\end{gathered}
$$

Here, $C_{c}$ is the Stokes-Cunningham coefficient, $K_{n}$ is the Knudsen number of particles, $\lambda$ is the free path of gas molecules.

(2) Thermophoretic Force

In a filed with a temperature gradient, small particles experience a force in the direction opposite to that of the gradient. We call this phenomenon as thermophoresis and the expression is shown below [12]:

$$
\begin{gathered}
F_{t h}=-D_{T, p} \frac{1}{m_{p} T} \nabla T \\
D_{T, p}=\frac{6 \pi d_{p} \mu^{2} C_{s}\left(K+C_{t} K_{n}\right)}{\rho_{f}\left(1+3 C_{m} K_{n}\right)\left(1+2 K+2 C_{t} K_{n}\right)}
\end{gathered}
$$

$D_{T, p}$ is the thermophoretic coefficient, $m_{p}$ is the particle mass, $T$ is the local fluid temperature. $K_{n}$ is the Knudsen number, $\lambda$ is the mean free path of the fluid, $K$ is the ratio of the fluid thermal conductivity $(k)$ and particle thermal conductivity $\left(k_{p}\right), C_{s}=1.17, C_{t}=2.18, C_{m}=1.14$.

(3) Saffman's lift force

The Saffman's lift force, or lift due to shear is caused by the difference of the particle velocity above and below. So the force direction is vertical to the particle velocity. The lift force used is from Li and Ahmadi and is a generalization of the expression provided by Saffman [13].

$$
F_{L}=\frac{2 K v^{1 / 2} \rho d_{i j}}{\rho_{p} D_{p}\left(d_{I k} d_{k I}\right)^{1 / 4}}\left(u_{f}-u_{p}\right)
$$

Where $K=2.594$ and $d_{i j}$ is the deformation tensor.

(4) Brownian Force

The components of the Brownian force are modeled as a Gaussian white noise process with spectral intensity $S_{n, i j}$ given by [14]:

$$
S_{n, i j}=S_{0} \delta_{i j}
$$

where $\delta_{i j}$ is the Kronecker delta function, and

$$
S_{0}=\frac{216 v k_{B} T}{\pi^{2} \rho_{f} D_{p}{ }^{5}\left(\frac{\rho_{p}}{\rho_{f}}\right)^{2} C_{c}}
$$

Here, $v$ is the kinematic viscosity, $T$ is the absolute temperature of the fluid, $C_{c}$ is the Cunningham correction, and $k_{B}$ is the Boltzmann constant.

Brownian force per unit particle mass are of the form:

$$
F_{B}(i)=\zeta_{i} \sqrt{\frac{\pi S_{0}}{\Delta t}}
$$

$\zeta_{i}$ are zero-mean, unit-variance-independent Gaussian random numbers and $\Delta t$ is the integral time step. For the randomness of Brownian force, it is necessary to calculate the value at every time step.

(5) Gravity and Buoyancy

For a spherical particle, the gravity and buoyancy are of the form

$$
\begin{gathered}
F_{g}=\pi D_{p}^{3} \rho_{p} g / 6 \\
F_{b u o}=\pi D_{p}^{3} \rho_{f} g / 6
\end{gathered}
$$

$g$ is the acceleration of gravity, usually considered to be $9.81 \mathrm{~m} / \mathrm{s}^{2}$.

For the purpose of this paper, the forces mentioned above are main forces acting on a particle in the corrugated pipe, other forces are assumed to be neglected in this paper.

\subsubsection{Fluctuating Velocity and Particles-Vortex Interaction}

In the stochastic tracking approach, velocity of the fluid can be expressed as the sum of average velocity $\bar{u}$ and fluctuating velocity $u$ to predict the dispersion of the particles due to turbulence [15].

$$
u=\bar{u}+u^{\prime}
$$

In the discrete random walk (DRW) model, the fluctuating velocity components are discrete piecewise constant functions of time. The value keeps constant over an interval of time determined by the characteristic lifetime of eddies in the field, so the DRW model can also be called as Eddy Life model.

There are two characteristic parameters of an eddy, which are fluctuating velocity $u$ following Gaussian distribution and eddy lifetime $\tau_{e}$.

$$
u^{\prime}=\zeta \sqrt{\overline{u^{\prime 2}}}
$$

$\zeta$ is a random number obeying the normal distribution and $\sqrt{\sqrt{u^{\prime 2}}}$ is the root mean square of fluctuating velocity.

For $k-\varepsilon$ model, $\sqrt{\overline{u^{\prime 2}}}$ can be presented as a function of turbulent kinetic energy:

$$
\sqrt{\overline{u^{\prime 2}}}=\sqrt{2 k / 3}
$$

$\tau_{e}$ represents the time an eddy exists and it can be defined as a constant [16]: 


$$
\tau_{e}=2 T_{L}
$$

The integral time $\left(T_{L}\right)$ for small "tracer" particles that move with the fluid is proportional to the particle dispersion rate.

For $k-\varepsilon$ model and its variants [17],

$$
T_{L} \approx 0.30 \frac{k}{\varepsilon}
$$

In order to determine the time that a particle interacts with an eddy, we must also get the time that a particle goes across an eddy,

$$
t_{\text {cross }}=-\tau \ln \left[1-\left(\frac{\mathrm{Le}}{\tau\left|u_{f}-u_{p}\right|}\right)\right]
$$

The particle is assumed to interact with the fluid phase eddy over the smaller of $\tau_{e}$ and $t_{\text {cross }}$ which is defined as $t_{\text {min }}$. In this time, instantaneous velocity is a constant. When this time is reached, a new value of the instantaneous velocity is obtained by applying a new value of $\zeta$.

\subsection{Integral Method}

The Pressure-Velocity Coupling method and SIMPLE arithmetic are applied to calculate the gas phase. For spatial discretization, second order upwind scheme is applied to calculate the pressure and momentum and first order upwind scheme is applied to calculate the turbulent kinetic energy and turbulent dissipation rate. The field is considered to be convergent when the residuals are less than $1 \times 10^{-6}$.

The integral time step is determined by two parameters: $t_{\min }$ and time step scale $\lambda$,

$$
\Delta t=\frac{t_{\min }}{\lambda}
$$

In this paper, $\lambda$ is set as a constant of 10 .

\section{Solvability Conditions and Verification}

\subsection{Solvability Conditions}

\subsubsection{Geometry and Meshing}

First of all, a typical corrugated tube is designed as the Figure below.

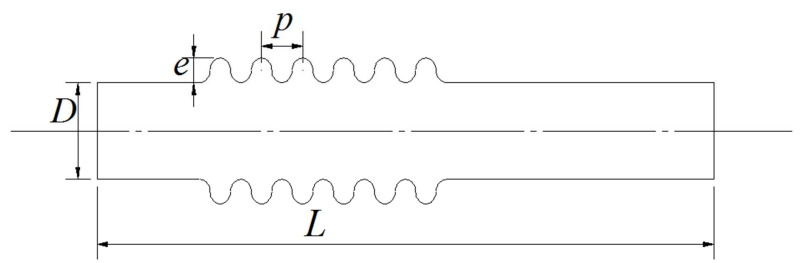

Figure 1. The structure of the corrugated tube.

In the Figure, $L$ is the length of the tube, in this paper it is a constant of $60 \mathrm{~mm} ; D$ is the inner diameter which is a constant of $8 \mathrm{~mm} ; e$ is the height of the tube and $p$ is the pitch of tube. In this paper, the variable of $e / p$ is selected to study the effect of structure parameter on the particle motion.

In order to simulate the movement of the fluid and particle near the wall, the "enhanced wall treatment" is applied. To ensure the calculation accuracy, the maximum element in the model is $0.01 \mathrm{~mm}$ and the minimum grid near the wall is set as $0.001 \mathrm{~mm}$. The total elements of tubes with different structure range from 272821 to 320750 . It has also been proved that the results of the cases with such number of elements are independent of the grid.

\subsubsection{Initial Conditions and Boundary Conditions}

In this paper, the fluid is incompressible and inlet velocity is considered to be known, so the velocity-inlet boundary conditions is selected. The pressure-outlet boundary conditions is applied at the exit. In the wall of the tube, no slip boundary condition is applied. The specification method of turbulence at the entrance is decided by setting values for turbulence intensity as $10 \%$ and hydraulic diameter as $20 \mathrm{~mm}$. In this paper, the inlet temperature is set as $800 \mathrm{~K}$, the outlet temperature is set as $450 \mathrm{~K}$ and the temperature of the wall is a constant of $350 \mathrm{~K}$.

At the entrance, the velocity of the fluid is given to the particles. During the calculation, the interaction between particles is ignored and particle's motion will have no effect on the fluid field, neither.

When a particle hits the wall, it is necessary to take the energy loss due to inelastic collision with the wall into consideration. In this paper, the particle rebounds off the boundary with a change in its momentum which can be defined as the coefficient of restitution.

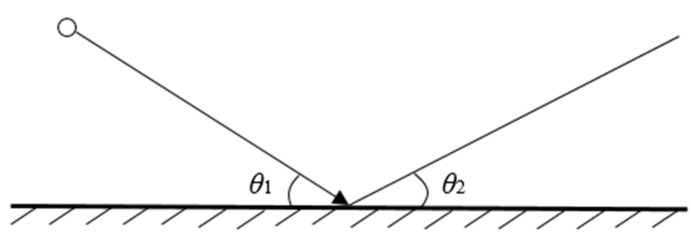

Figure 2. Particle reflection at wall.

The reflection coefficient, or the coefficient of restitution defines the rest of momentum after the collision with the wall. For example in the normal direction:

$$
e_{n}=\frac{v_{2 n}}{v_{1 n}}
$$

Here, $v_{1 n}$ and $v_{2 n}$ are the particle velocity normal to the wall before and after collision. In the direction tangential to the wall, the coefficient is defined in the same way. Li and Ahmadi confirmed the mechanism of collision acting on the particle and found that the influence of collision mainly plays an important role in particles with larger size. Usually the coefficient was conducted by experiment, in this paper, the reflection coefficient in the normal and tangential direction is defined as follows [5]: 


$$
\begin{gathered}
e_{n}=\frac{v_{2 n}}{v_{1 n}}=1.0-0.4159 \theta_{1}-0.4994 \theta_{1}^{2}+0.292 \theta_{1}^{3} \\
e_{t}=\frac{v_{2 t}}{v_{1 t}}=1.0-2.12 \theta_{1}+3.0775 \theta_{1}^{2}-1.1 \theta_{1}^{3}
\end{gathered}
$$

Here, $e_{n}$ is the coefficient of restitution in the normal direction, $e_{t}$ is the coefficient of restitution in the tangential direction. $\theta$ is the angle of incidence.

\subsection{Verification}

Simulation results of the particle deposition are usually given by deposition rate of the form [18]:

$$
V_{d}=\frac{J}{C_{0}}
$$

Here, $J$ is the mass flow settling to the wall per unit time. $C_{0}$ is the average particle concentration.

Based on the number of particles at entrance and exit, a more convenient method is put forward to calculate the deposition rate [19]:

$$
V_{d}=-\frac{D u_{\text {mean }} \ln \left(N_{P, \text { out }} / N_{P, \text { in }}\right)}{L}
$$

Here, $D$ and $L$ are the diameter and length of the tube. $u_{\text {mean }}$ is the axial average flow velocity. $N_{P, \text { in }}$ and $N_{P, \text { out }}$ are number of particles at entrance and exit. The dimensionless deposition velocity $V_{d}^{+}$is defined by normalizing the deposition velocity with the friction $u^{*}$.

$$
V_{d}^{+}=V_{d} / u^{*}
$$

The dimensionless particle relaxation time $\tau_{p}^{+}$is used here to evaluate particle deposition time in turbulence flow. $\tau_{p}{ }^{+}$can be calculated by the equation as follows:

$$
\tau_{p}^{+}=\frac{\tau_{p} u^{* 2}}{v}=S d_{p}^{2} C_{c} u^{* 2} / 18 v^{2}=C_{c} \rho_{p} d_{p}^{2} u^{* 2} / 18 \mu v
$$

To prove the correctness of the numerical model, the dimensionless deposition velocity with different dimensionless particle relaxation time has been studied numerically in a tube which has the same geometry and setting as a reference published by Fan and Ahdami [20].

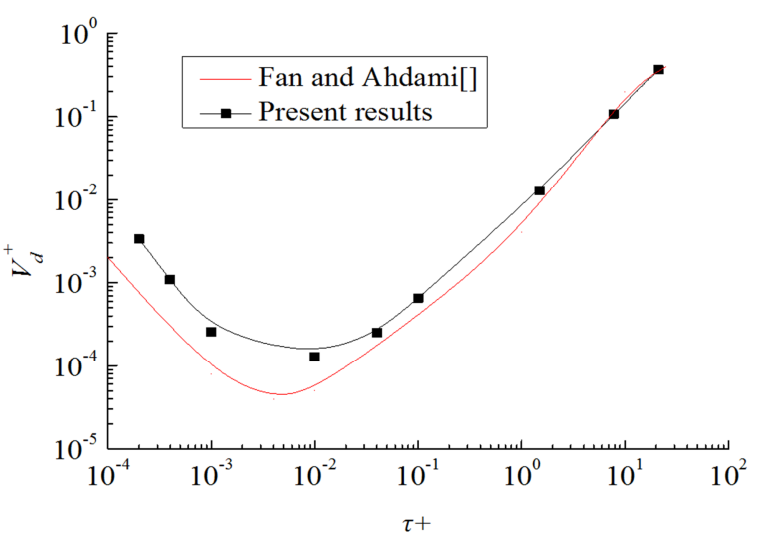

Figure 3. Numerical results compared with Fan and Ahdami.

As Figure 3 shows, the result of dimensionless deposition velocity $V_{d}^{+}$with different dimensionless particle relaxation time $\tau_{p}^{+}$fits in well with that calculated by Fan and Ahdami [19], especially for particles with lager diameters. For micro particles, the assumption of isotropy in the $k-\varepsilon$ turbulence model leads to high level of fluctuation perpendicular to the wall and higher prediction of deposition rates. In this paper, the trajectories and deposition rates of particles with different conditions are discussed. So the model is considered to be able to calculate the particle motion in a corrugated tube.

\section{The Trajectories of Particles in Different Conditions}

In this section, the numerical methods and solvability conditions are applied to calculated the trajectories of particles in the corrugated tube. It should be mentioned that variables of $e / p$ and inlet velocity are selected to study on influencing factor of the particle trajectory.

\subsection{Particle Trajectories with Different Released Position}

In an EGR valve, the valve lift controls the amount of gas returns to the cylinder. Obviously, particles in EGR valves with different lift will show different transfer characteristics. The 100 particles are released from each location, and 5 representative trajectories are selected for analysis. In order to study the differences between trajectories of different particle sizes, 10 micron, 1 micron and 0.1 micron particles are selected.

Figure 4 shows the trajectories of $10 \mu \mathrm{m}$ particles released from $0.1 \mathrm{~mm}, 0.5 \mathrm{~mm}$ and $1 \mathrm{~mm}$ near the wall. It should be mentioned that in these cases, $e=2 \mathrm{~mm}, p=4 \mathrm{~mm}$, inlet velocity $=30 \mathrm{~m} / \mathrm{s}$, inlet temperature $=800 \mathrm{~K}$.

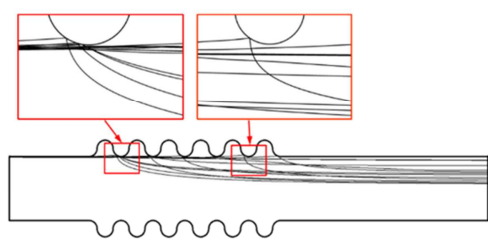

(a) released from $0.1 \mathrm{~mm}$ near the wall

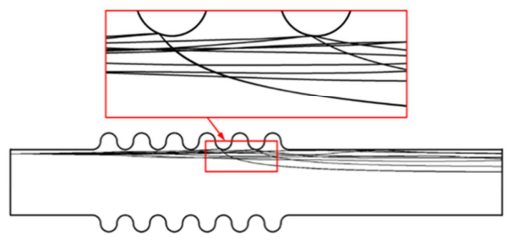

(b) released from $0.5 \mathrm{~mm}$ near the wall

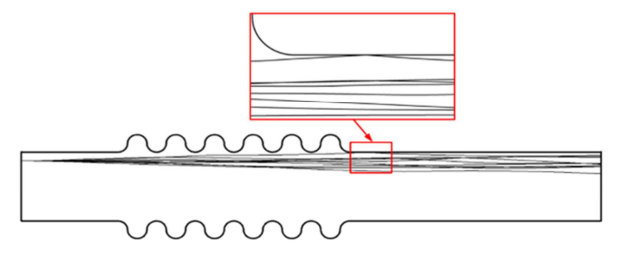

(c) released from $1 \mathrm{~mm}$ near the wall

Figure 4. The trajectories of $10 \mu$ m particles. 
As can be seen from Figure 4, the positions where $10 \mu \mathrm{m}$ particles hit the wall are always on the left side of the trough and very close to the trough. Particles that hit the left side of the trough always bound back into the main stream and gradually become consistent with the airflow velocity in the main stream. No particles bounce back into the node of the pipe. The farther the release position of particles is from the wall, the farther the particles collide with the wall.

Figure 5 shows the trajectories of $1 \mu \mathrm{m}$ particles released from $0.1 \mathrm{~mm}, 0.5 \mathrm{~mm}$ and $1 \mathrm{~mm}$ near the wall. It should be mentioned that in these cases, $e=2 \mathrm{~mm}, p=4 \mathrm{~mm}$, inlet velocity $=30 \mathrm{~m} / \mathrm{s}$, inlet temperature $=800 \mathrm{~K}$.

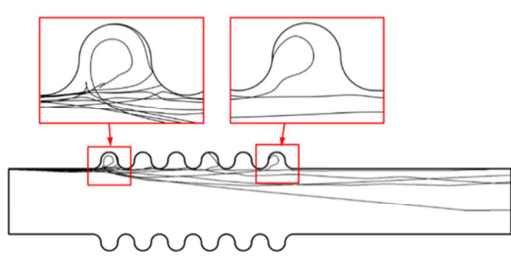

(a) released from $0.1 \mathrm{~mm}$ near the wall

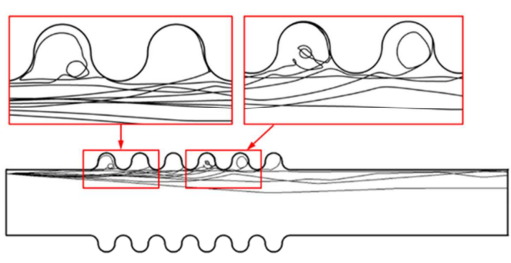

(b) released from $0.5 \mathrm{~mm}$ near the wall

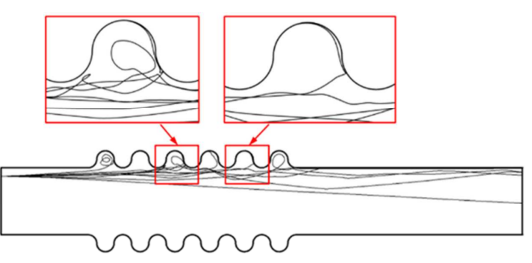

(c) released from $1 \mathrm{~mm}$ near the wall

Figure 5. The trajectories of $1 \mu \mathrm{m}$ particles.

As can be seen from Figure 5, 1um particles may enter the node, and the farther away the particle release position is from the wall, the farther the particle enters the node. However, there is no necessary relationship between the particle trajectory after entering the node and the particle release position. After entering the node, the particles move counterclockwise, either colliding into the wall surface under inertia, or following the fluid to flow around the node.

Figure 6 shows the trajectories of $0.1 \mu \mathrm{m}$ particles released from $0.1 \mathrm{~mm}, 0.5 \mathrm{~mm}$ and $1 \mathrm{~mm}$ near the wall. It should be mentioned that in these cases, $e=2 \mathrm{~mm}, p=4 \mathrm{~mm}$, inlet velocity $=30 \mathrm{~m} / \mathrm{s}$, inlet temperature $=800 \mathrm{~K}$.

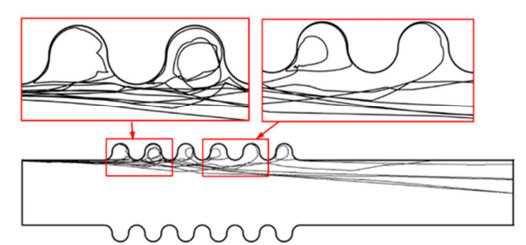

(a) released from $0.1 \mathrm{~mm}$ near the wall

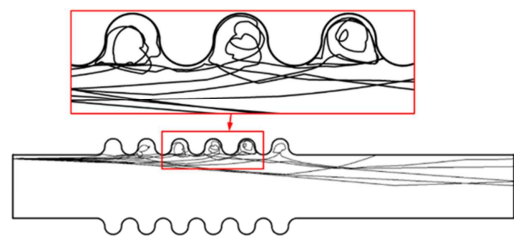

(b) released from $0.5 \mathrm{~mm}$ near the wall

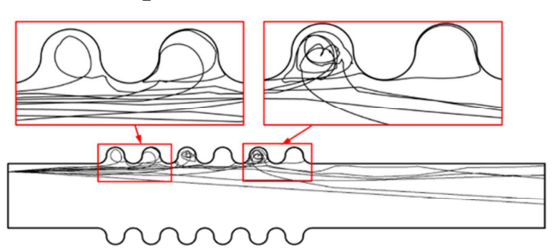

(c) released from $1 \mathrm{~mm}$ near the wall

Figure 6. The trajectories of $0.1 \mu$ m particles.

As can be seen from Figure 6, the retention time of $0.1 \mu \mathrm{m}$ particles in the node is very long. It is an evident phenomenon that particles move with the fluid, and particles move randomly under the turbulent fluctuation. $0.1 \mu \mathrm{m}$ particles also move counterclockwise within the nodes, but the number of particles touching the wall is less than that of the $1 \mu \mathrm{m}$ ones.

\subsection{Particle Trajectories with Different e/p of the Corrugated Pipe}

Different $e / p$ means different structures of the corrugated

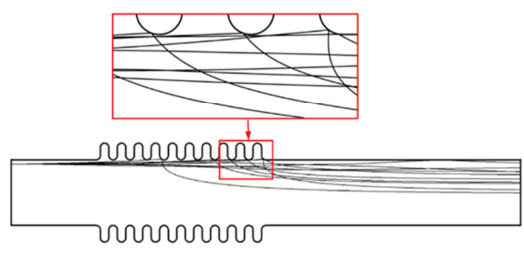

(a) $e / p=2 / 2$

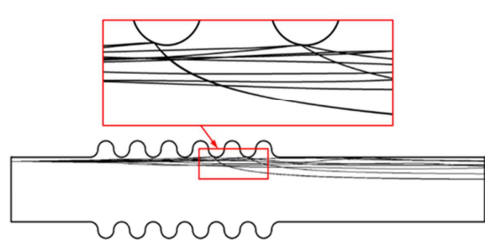

(b) $e / p=2 / 4$ pipe. The structure determines the distribution of flow field, as well as the trajectory of the particle. In the following analysis, $e / p$ of $2 / 2,2 / 4$ and $2 / 6$ are chosen to study the effect of structure on particle trajectory. It should be mentioned that in these cases, the particles are released $0.5 \mathrm{~mm}$ from the wall, inlet velocity $=30 \mathrm{~m} / \mathrm{s}$, inlet temperature $=800 \mathrm{~K}$.

Figure 7 shows the trajectories of $10 \mu \mathrm{m}$ particles in the corrugated pipe with $e / p$ of $2 / 2,2 / 4$ and $2 / 6$.

Figure 7. The trajectories of $10 \mu \mathrm{m}$ particles with different e/p.

As can be seen from Figure 7, $10 \mu \mathrm{m}$ particles cannot go inside the node of the corrugated pipe, so the structure of the pipe will not make difference to the trajectory of $10 \mu \mathrm{m}$ particles.

Figure 8 shows the trajectories of $1 \mu \mathrm{m}$ particles in the corrugated pipe with $e / p$ of $2 / 2,2 / 4$ and $2 / 6$. 


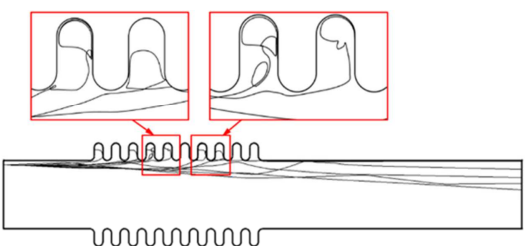

(a) $e / p=1 / 1$

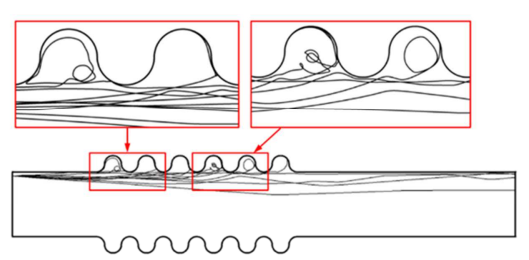

(b) $e / p=\mathbf{1} / 2$

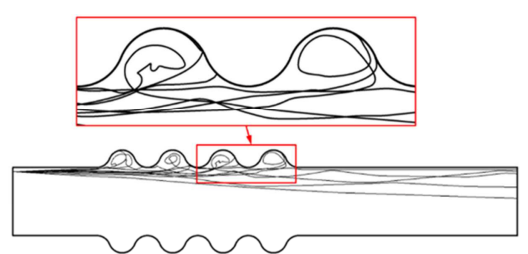

(c) $e / p=1 / 3$

Figure 8. The trajectories of $1 \mu \mathrm{m}$ particles with different e/p.

As can be seen from Figure 8 , when $e / p$ is less than $1 / 2$, the particle trajectory is relatively simple. There are some particles colliding into the wall under inertia, and others flowing around the node with the fluid. The trajectories are similar to the shape of the node. When $e / p$ is larger than $1 / 2$, the trajectories are divided into two parts: counterclockwise motion in the lower part and clockwise motion in the upper part.

Figure 9 shows the trajectories of $0.1 \mu \mathrm{m}$ particles in the corrugated pipe with $e / p$ of $2 / 2,2 / 4$ and $2 / 6$.

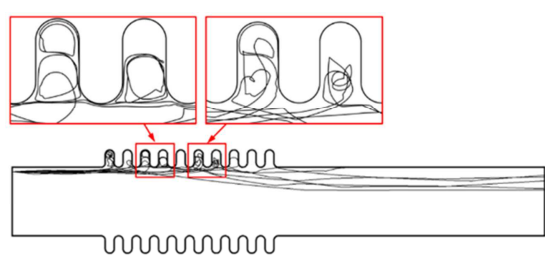

(a) $e / p=1 / 1$

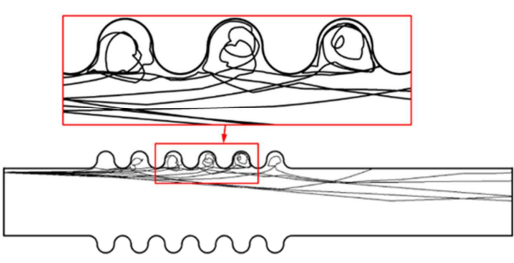

(b) $e / p=1 / 2$

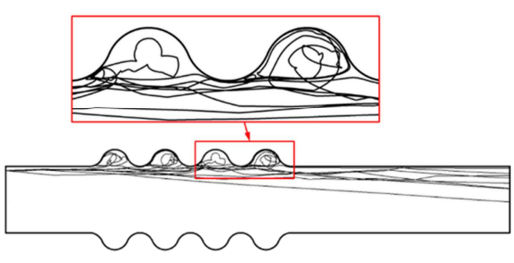

(c) $e / p=\mathbf{1} / \mathbf{3}$

Figure 9. The trajectories of 0.1 $\mu$ m particles with different e/p.

As can be seen from Figure 9, when $e / p$ is less than $1 / 2$, the retention time of particles is longer, so the trajectories fits the shape of nodes very well. When $e / p$ is larger than $1 / 2$, the particles move counterclockwise in the lower part and clockwise in the upper part. And the velocity of the particle in the upper part is very small, making it easier for the particle to deposit on the wall.

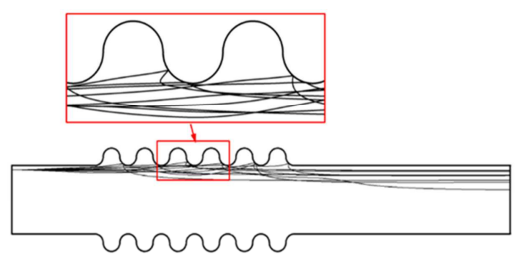

(a) $v=10 \mathrm{~m} / \mathrm{s}$

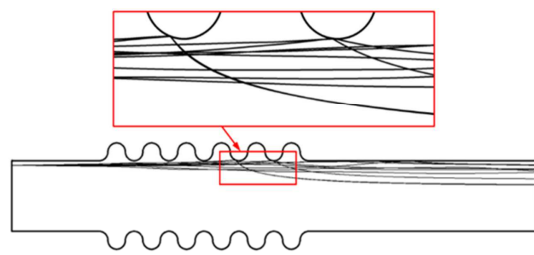

(b) $v=30 \mathrm{~m} / \mathrm{s}$

\subsection{Particle Trajectories with Different Inlet Velocity}

In the following analysis, inlet velocity of $10 \mathrm{~m} / \mathrm{s}, 30 \mathrm{~m} / \mathrm{s}$ and $50 \mathrm{~m} / \mathrm{s}$ are chosen to study the effect of inlet velocity on particle trajectory. It should be mentioned that in these cases, the particles are released $0.5 \mathrm{~mm}$ from the wall, $e=2 \mathrm{~mm}$, $p=4 \mathrm{~mm}$, inlet temperature $=800 \mathrm{~K}$.

Figure 10 shows the trajectories of $10 \mu \mathrm{m}$ particles in the corrugated pipe with inlet velocity of $10 \mathrm{~m} / \mathrm{s}, 30 \mathrm{~m} / \mathrm{s}$ and $50 \mathrm{~m} / \mathrm{s}$.

Figure 10. The trajectories of $10 \mu \mathrm{m}$ particles with different inlet velocity.

As can be seen in Figure 10, when inlet velocity increases, the $10 \mu \mathrm{m}$ particles will show worse randomness caused by turbulent kinetic energy, so it will be more difficult for the

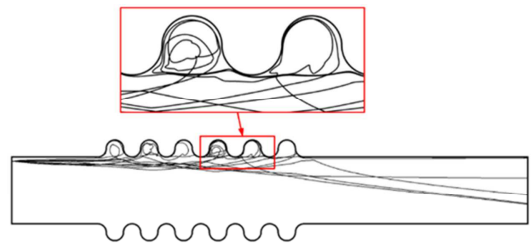

(a) $v=10 \mathrm{~m} / \mathrm{s}$

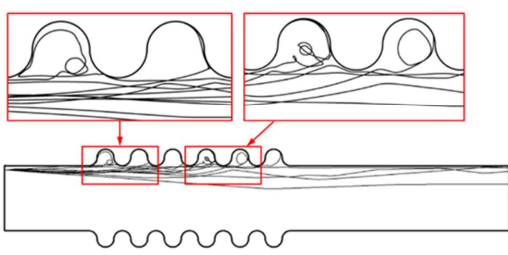

(b) $v=30 \mathrm{~m} / \mathrm{s}$

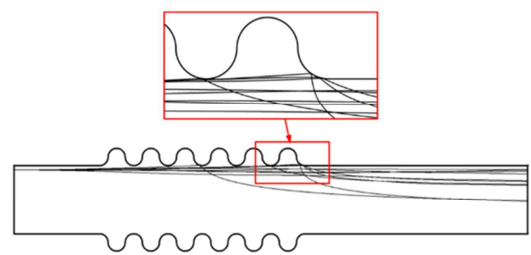

(c) $v=50 \mathrm{~m} / \mathrm{s}$ particle to go inside the node.

Figure 11 shows the trajectories of $1 \mu \mathrm{m}$ particles in the corrugated pipe with inlet velocity of $10 \mathrm{~m} / \mathrm{s}, 30 \mathrm{~m} / \mathrm{s}$ and $50 \mathrm{~m} / \mathrm{s}$.

Figure 11. The trajectories of $1 \mu \mathrm{m}$ particles with different inlet velocity.

As can be seen in Figure 11, inlet velocity will make obvious effect on the trajectories of $1 \mu \mathrm{m}$ particles. When the inlet velocity is $10 \mathrm{~m} / \mathrm{s}$, the particle will move with the fluid very well, and the time that particle moving inside the node is longer. When the inlet velocity is $50 \mathrm{~m} / \mathrm{s}$, the particle will soon collide to the wall. So the larger is the inlet velocity, the better 
inertial effect the particle will show.

Figure 12 shows the trajectories of $0.1 \mu \mathrm{m}$ particles in the

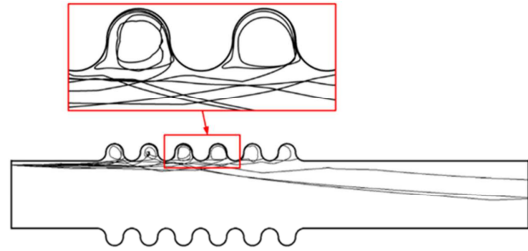

(a) $v=10 \mathrm{~m} / \mathrm{s}$

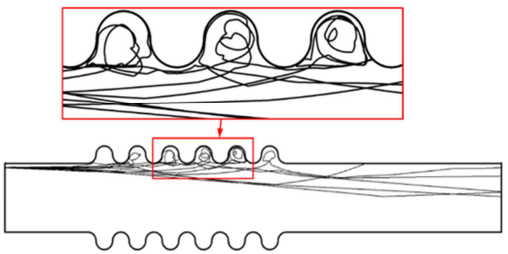

(b) $v=30 \mathrm{~m} / \mathrm{s}$

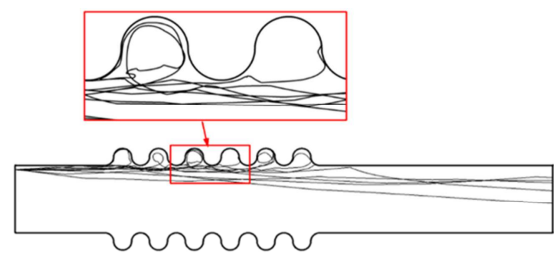

(c) $v=50 \mathrm{~m} / \mathrm{s}$

Figure 12. The trajectories of $0.1 \mu \mathrm{m}$ particles with different inlet velocity.

As can be seen from Figure 12, no matter how the inlet velocity varies from $10 \mathrm{~m} / \mathrm{s}$ to $50 \mathrm{~m} / \mathrm{s}$, the $0.1 \mu \mathrm{m}$ particles follow the fluid very well. So the effect of inlet velocity on $0.1 \mu \mathrm{m}$ particles is not so great as that on $1 \mu \mathrm{m}$ ones.

\section{Conclusion}

In this paper, the author used the DRW model to calculated the particle trajectory in the corrugated pipe, especially in the node. On the basis, the author also analysis the influence of structure parameters and inlet velocity on particle trajectory, the following conclusions are acquired:

(1) The farther away the particle is released from the wall, the farther the particle collides with the wall. But the trajectory after the particle entering the node has nothing to do with the release position.

(2) The $10 \mu \mathrm{m}$ particles has good inertia and cannot enter the node. $1 \mu \mathrm{m}$ particles may not only collide into the wall under inertia, but also move counterclockwise within the node with the fluid. $0.1 \mu \mathrm{m}$ particles have a good following quality, and have a longer residence time in the node and a longer trajectory.

(3) The parameter of $e / p$ has a great influence on the trajectory of the particles entering the node. When $e / p$ is less than $1 / 2$, the trajectories are similar to the shape of the nodes. When $e / p$ is greater than $1 / 2$, the trajectories of particles in the nodes can be divided into two parts: the lower part moves counterclockwise, the upper part moves clockwise. And the velocity of the upper part is very small, so it is easy for the particle to deposit on the wall.

(4) The larger the inlet velocity is, the stronger inertia particles' trajectory will show. For particles with moderate size, the influence of inlet velocity on particle trajectory is crucial.

\section{Acknowledgements}

This study was funded by the National Natural Science Foundation of China (Grant No. 51776016, 51606006), Beijing Natural Science Foundation (Grant No. 3172025,3182030), National Key Research and Development Program (Grant No. 2017YFB0103401), National Engineering Laboratory for Mobile Source Emission Control Technology (Grant No. NELMS2017A10), and the Talents
Foundation of Beijing Jiaotong University (Grant No. 2018RC017).

\section{References}

[1] Hinds, W. C. Properties, behavior, and measurement of airborne Particles [J]. Aerosol technology, 1984.

[2] Hidy, G. M. an industrial and environmental science [J]. Aerosols, 1984

[3] Papavergos, P. G. \& Hedley, A. B. Particle deposition behaviour from turbulence flows $[\mathrm{J}]$. Chemical Engineering Research and Design, 1984, 62, 275-295.

[4] McLaughlin J. B. Aerosol particle deposition in numerically simulated channel flow [J]. Phys. Fluids A, 1989, 1 (7): $1211 \sim 1224$

[5] Fan, F. G. \& Ahmadi, G. A sublayer model for turbulent deposition of particles in vertical ducts with smooth and rough surfaces [J]. Journal of Aerosol Science, 24, 45-64.

[6] Thakurta, D. G. Chen, McLaughlin J. B, Kontomaris K. Thermophoretic deposition of small particle in a direct numerical simulation of turbulent channel flow. International journal of heat and mass transfer [J] 1998, 41: 4167-4182.

[7] Gosman, A. D. Ioannides, E. Aspects of computer simulation of liquid fuelled combustors [J]. J. Energy 7, 482-490.

[8] Kallio G. A., Reeks M. W. A numerical simulation of particle deposition in turbulent boundary layers [J]. International Journal of Multiphase Flow, 15 (3): 433-446.

[9] Hao Lu, Lin Lu. Numerical investigation on particle deposition enhancement in duct air flow by ribbed wall [J]. Building and Environment, 2015, 85: 61-72.

[10] Bai Zhenxiao. Analysis of the Diesel Exhaust Particle Transport and Deposition in Turbulent Flow [D]. Beijing: Beijing Jiaotong University, 2011.

[11] A. D. Gosman, E. Ioannides. Aspects of computer simulation of liquid-fuelled combustors. [J]. Energy, 1983, 7 (6): 482-490.

[12] L. Talbot et al. Thermophoresis of Particles in a Heated Boundary Layer. [J]. Fluid Mech, 1980, 101 (4): 737-758.

[13] P. G. Saffman. The Lift on a Small Sphere in a Slow Shear Flow. [J]. Fluid Mech, 1965, 22: 385-400.

[14] A. Li, G. Ahmadi. Dispersion and Deposition of Spherical Particles from Point Sources in a Turbulent Channel Flow. [J]. Aerosol Science and Technology, 1992, 16: 209-226. 
[15] Q. Zhou and M. A. Leschziner. Technical report [C]// 8th Turbulent Shear Flows Symp, Munich. 1991.

[16] Matida Edgar Akio, Nishino Koichi, Torii Kahoru. Statistical simulation of particle deposition on the wall from turbulent dispersed pipe flow [J]. International Journal of Heat and Fluid Flow, 2000, 21 (4): 389-402.

[17] B. J. Daly and F. H. Harlow. Transport Equations in Turbulence [J]. Phys. Fluids. 1970, 13: 2634-2649.
[18] Zhang Z, Chen Q. Prediction of particle deposition onto indoor surfaces by CFD with a modified Lagrangian method [J]. Atmospheric Environment, 2009, 43 (2): 319-328.

[19] Zhang Jinping, Li Angui, Li Desheng. Modeling deposition of particles in typical horizontal ventilation duct flows [J]. Energy Conversion and Management, 2008, 49 (12): 3672-3683.

[20] Fan, F. G. \& Ahmadi, G. A sublayer model for turbulent deposition of particles in vertical ducts with smooth and rough surfaces [J]. Journal of Aerosol Science, 2007, 24: 45-64. 